\title{
Pesquisa fenotípica dos fatores de virulência em Pseudomonas aeruginosa isolados de água de abastecimento público
}

Phenotypic research of virulence factors in Pseudomonas aeruginosa isolated from public water supply

\author{
E. K. Saeki ${ }^{1 *}$; A. Y. Yamada ${ }^{1}$ I. A. de Souza ${ }^{1}$; L. A. de Araujo ${ }^{1}$; L. Anversa ${ }^{2}$; R. \\ K. T. Kobayashi ${ }^{3}$; G. Nakazato ${ }^{3}$ \\ ${ }^{1}$ Centro de Laboratório Regional, Instituto Adolfo Lutz, 19013-050, Presidente Prudente-SP, Brasil \\ ${ }^{2}$ Centro de Laboratório Regional, Instituto Adolfo Lutz, 17015-110, Bauru-SP, Brasil \\ ${ }^{3}$ Laboratório de Bacteriologia Básica e Aplicada/Centro de Ciências Biológicas, Universidade Estadual de \\ Londrina, 86057-970, Londrina-PR, Brasil \\ *erika.saeki@ial.sp.gov.br
}

(Recebido em 07 de maio de 2020; aceito em 05 de junho de 2020)

\begin{abstract}
A bactéria Pseudomonas aeruginosa está amplamente distribuída no ambiente. A pouca exigência nutricional deste microrganismo possibilita sua sobrevivência em água de abastecimento público, cuja importância para a saúde pública deve-se principalmente ao fato de causar infecções em indivíduos imunocomprometidos. O objetivo deste trabalho foi avaliar fenotipicamente os fatores de virulência de $P$. aeruginosa obtidos de água de abastecimento público. Um total de 19 isolados de $P$. aeruginosa foram analisados para determinação das motilidades (swarming, swimming e twitching), e avaliação da produção de protease alcalina, ramnolipídeos e DNase. Os experimentos foram realizados em triplicata em três ocasiões distintas. Para realizar a representação gráfica e a análise estatística foi utilizado o Software R Studio (1.2.5001). Todos os isolados foram produtores de protease alcalina com halo de proteólise acima de $8 \mathrm{~mm}$. A atividade da DNase e a produção de ramnolipídeos foram observadas em $21,1 \%(\mathrm{n}=4)$ e $84,2 \%(n=16)$, respectivamente. Os resultados mostraram uma alta diversidade no comportamento de motilidade entre os isolados de $P$. aeruginosa. Ao comparar os três tipos de motilidade, a maioria dos isolados $(68,4 \%)$ apresentou produção significativa $(\mathrm{p}<0,05)$ de motilidade swimming. Conclui-se que os isolados de $P$. aeruginosa testados foram capazes de produzir fatores de virulência como motilidade swimming e twitching, protease e ramnolipídeos.

Palavras-chave: DNases, qualidade da água, motilidade.
\end{abstract}

The bacterium Pseudomonas aeruginosa is widely distributed in the environment. The low nutritional requirement of this microorganism makes it possible to survive in public water supply, whose importance for public health is mainly due to the fact that it causes infections in immunocompromised individuals. The objective of this work was evaluate phenotypically the virulence factors of $P$. aeruginosa obtained from public water supply. A total of $19 P$. aeruginosa isolates were analyzed to determine motilities (swarming, swimming, and twitching), and to evaluate the production of alkaline protease, rhamnolipids, and DNase. The experiments were carried out in triplicate on three different occasions. The Software R Studio (1.2.5001) was used to perform the graphical representation and the statistical analysis. All isolates were producers of alkaline protease with a proteolysis halo above $8 \mathrm{~mm}$. DNase activity and rhamnolipids production were observed in $21.1 \%(n=4)$ and $84.2 \%(n=16)$, respectively. The results showed a high diversity in motility behavior among $P$. aeruginosa isolates. When comparing the three types of motility, most isolates $(68.4 \%)$ showed significant production $(\mathrm{p}<0.05)$ of swimming motility. The results showed the $P$. aeruginosa isolates tested were able to produce virulence factors such as swimming and twitching motility, protease and rhamnolipids.

Keywords: DNases, water quality, motility.

\section{INTRODUÇÃO}

Pseudomonas aeruginosa é uma bactéria Gram-negativa amplamente distribuída no ambiente, presente no solo, na água e na matéria orgânica em decomposição. É frequentemente responsável por infecções no trato urinário e respiratório, podendo evoluir para sepse, 
principalmente em portadores de fibrose cística e pacientes imunocomprometidos ou com extensas queimaduras na pele $[1,2]$. É o agente mais comum em infecções relacionadas à assistência à saúde (IRAS), associada com significativa taxa de morbi-mortalidade devido à capacidade do microrganismo de desenvolver rapidamente resistência a antibióticos, adaptar-se facilmente às mudanças no ambiente, e expressar uma variedade de fatores de virulência, como motilidade, formação de biofilme, síntese de protease alcalina, de ramnolipídeos e de DNases [3].

$P$. aeruginosa utiliza estruturas de superfície para realização da motilidade, sendo caracterizada por três tipos de movimentos, denominados, swarming, swimming e twitching, expressos de acordo com a viscosidade do meio [4]. As propriedades da superfície a ser colonizada, o arranjo de pili na superfície bacteriana, e as condições ambientais como concentração de oxigênio e o fluxo de fluido, são fatores que influenciam essas motilidades [5].

Swarming é a movimentação bacteriana que ocorre através da ação conjunta e organizada de inúmeras bactérias, através de um substrato semi-sólido, dependente de flagelo em colaboração com a pili tipo IV [6]. Na motilidade swimming são necessárias as mesmas estruturas para locomoção, no entanto, as bactérias movimentam-se individualmente ao longo da superfície de uma camada aquosa [7]. Na motilidade twitching é utilizada somente a pili tipo IV para o deslocamento, que ocorre através de movimentos repetitivos de retração e extensão em uma superfície sólida [6].

Com a expressão dos diferentes tipos de motilidade, a bactéria é capaz de formar biofilmes que possibilitam a persistência do microrganismo em sistemas de abastecimento de água por longos períodos [8]. As saídas de água, como as torneiras, são possíveis reservatórios desses biofilmes, e sua presença no ambiente hospitalar merece atenção [9].

A protease alcalina tem papel importante na fuga fagocitária e é capaz de degradar um amplo número de proteínas do hospedeiro, incluindo a fibronectina e a laminina, facilitando no processo de invasão da bactéria [10]. Os ramnolipídeos são biosurfactantes que auxiliam nos estágios iniciais da formação de biofilmes e tem papel na regulação da motilidade [11]. A DNase é outro fator de virulência que auxilia a bactéria na fuga de neutrófilos ligados a captura e eliminação de invasores [12]. Desta forma, bactérias como $P$. aeruginosa produtoras de diversos fatores de virulência podem estar presentes no ambiente, inclusive em sistemas de abastecimento de água [2].

Sabe-se que a qualidade da água afeta direta e indiretamente a saúde das pessoas. A água de abastecimento contaminada por microrganismos pode gerar surtos de doenças como cólera e outras infecções gastrointestinais, às vezes afetando milhares de pessoas e causando muitas mortes, por isso, seu tratamento e distribuição adequados são de fundamental relevância [13]. No Brasil, além de receber o tratamento, a água deve atender ao padrão de potabilidade estabelecido pela Portaria de Consolidação n ${ }^{\circ} 5$ de 28 de setembro de 2017, na qual determina a pesquisa de coliformes totais e Escherichia coli como indicadores de qualidade microbiológica [14]. No entanto, esta Portaria não expressa valores para $P$. aeruginosa [15]. A presença dessa bactéria em água tratada gera preocupação pelo fato de ser responsável por infecções crônicas em portadores de fibrose cística, o que resultam em prognósticos ruins aos pacientes e mudança de estilo de vida para que evitem exposição a $P$. aeruginosa, além de poder comprometer a saúde de pessoas de outros grupos de risco [16].

Por isso, a avaliação da virulência de $P$. aeruginosa presente em água se faz necessária para obtermos conhecimento sobre a patogenicidade desses microrganismos. O objetivo deste estudo foi avaliar a expressão fenotípica dos fatores de virulência de $P$. aeruginosa isolados de água de abastecimento público.

\section{MATERIAL E MÉTODOS}

\subsection{Isolados bacterianos e área de estudo}

Um total de 19 isolados de Pseudomonas aeruginosa foram obtidos de amostras de água dos sistemas de abastecimento público no centro-oeste do estado de São Paulo no ano de 2016 [2]. $P$. aeruginosa foram isolados pela técnica de membrana filtrante em ágar m-PA-C e 
identificados pela coloração de Gram, morfologia da colônia, crescimento em ágar cetrimide, catalase positiva, oxidase positiva e não fermentador de glicose.

As culturas de estoque de cada isolado foram mantidas por curto prazo à temperatura ambiente em ágar estoque. Para armazenamento em período mais longo, os isolados foram congelados a $-20{ }^{\circ} \mathrm{C}$ em caldo Luria-Bertani (LB, Neogen) com $20 \%$ de glicerol (v / v, Merck).

Os fatores de virulência foram pesquisados no Centro de Laboratório Regional do Instituto Adolfo Lutz de Presidente Prudente-V (CLR-IAL-PP-V) entre os anos de 2018 e 2019. A cepa $P$. aeruginosa $\mathrm{PAO}$ foi utilizada como padrão de referência, por esta cepa ser caracterizada como produtora de motilidade, protease alcalina e ramnolipídeos.

\subsection{Fatores de virulência}

Motilidade swarming. Um volume de $10 \mu \mathrm{L}$ de culturas previamente crescidas a $37^{\circ} \mathrm{C}$ por $24 \mathrm{~h}$ em caldo LB (Neogen) foram inoculadas no centro do ágar Swarming (Glicose 1,0\%; Peptona $0,5 \%$; Extrato de levedura 0,2\%; Ágar 0,5\%). As placas foram incubadas sem inverter a posição a $30^{\circ} \mathrm{C}$ por $24 \mathrm{~h}$ [17]. As médias dos diâmetros (d) das motilidades foram calculadas e categorizadas de acordo com Abdouchakour et al. (2018) [18]: não móvel ou fracamente móvel d $<20 \mathrm{~mm}$; moderadamente móvel $20 \mathrm{~mm} \leq \mathrm{d}<40 \mathrm{~mm}$; e altamente móvel d $\geq 40 \mathrm{~mm}$.

Motilidade swimming. Uma colônia de cada isolado foi inoculada em superfície do ágar Swimming (Triptona 1,0\%; $\mathrm{NaCl} \mathrm{0,5 \% ;} \mathrm{Ágar} \mathrm{0,3 \% ).} \mathrm{As} \mathrm{placas} \mathrm{foram} \mathrm{incubadas} \mathrm{sem} \mathrm{inverter} \mathrm{a}$ posição a $30^{\circ} \mathrm{C}$ por $24 \mathrm{~h}$ [19]. As médias dos diâmetros das motilidades (d) foram calculadas e categorizadas de acordo com Abdouchakour et al. (2018) [18]: não móvel ou fracamente móvel $\mathrm{d}<20 \mathrm{~mm}$; moderadamente móvel $20 \mathrm{~mm} \leq \mathrm{d}<40 \mathrm{~mm}$; e altamente móvel $\mathrm{d} \geq 40 \mathrm{~mm}$.

Motilidade twitching. Uma colônia de cada isolado foi inoculada até o fundo da placa de ágar Twitching (Triptona 1,0\%; Extrato de levedura 0,5\%; $\mathrm{NaCl} 1,0 \%$; Ágar 1,0\%). Após incubação a $37^{\circ} \mathrm{C}$ por $24 \mathrm{~h}$, o ágar foi cuidadosamente removido e a zona de motilidade foi medida pela coloração da placa de Petri com cristal violeta $2 \%$ por $2 \mathrm{~h}$ [6]. Como controle negativo, cada isolado foi inoculado em placa com ágar Triptona de soja (TSA, Difco) sob as mesmas condições dos testes. As médias das zonas de motilidade foram calculadas e categorizadas de acordo com Wolska e Kot (2013) [20]: não móvel d < 7; fracamente móvel 7 $\mathrm{mm}<\mathrm{d}<15 \mathrm{~mm}$; moderadamente móvel $15 \mathrm{~mm}<\mathrm{d}<25 \mathrm{~mm}$; e altamente móvel d $>25 \mathrm{~mm}$.

Protease alcalina. A produção de protease alcalina foi determinada em placas de ágar Leite (Leite em pó 1,0\%; Peptona 0,1\%; $\mathrm{NaCl} 0,5 \%$; Ágar 2,0\%; $\mathrm{pH} 10,0$ ). Um volume de $10 \mu \mathrm{L}$ das células em suspensão de $P$. aeruginosa de cada cepa foi adicionado à placa de ágar Leite e incubado a $37^{\circ} \mathrm{C}$ por $24 \mathrm{~h}$. A produção de protease alcalina foi confirmada pela formação de uma zona clara ao redor do inóculo bacteriano, o diâmetro do halo foi medido e classificado em: +++ $(8-25 \mathrm{~mm}) ;++(5-8 \mathrm{~mm}) ;+(1-5 \mathrm{~mm}) ; \mathrm{e}-(0 \mathrm{~mm})$.

Ramnolipídeos. A produção de ramnolipídeos foi estimada utilizando ensaios em placas de ágar CTAB (Brometo de cetiltrimetilamônio), conforme Siegmund e Wagner (1991) [21]. Um volume de $10 \mu \mathrm{L}$ de cada isolado de $P$. aeruginosa crescidos em caldo LB (Neogen) a $37^{\circ} \mathrm{C}$ por $24 \mathrm{~h}$ foram inoculados nos poços confeccionados nas placas e incubados a $37^{\circ} \mathrm{C}$ por $48 \mathrm{~h}$. A indicação da produção de ramnolipídeos ocorreu pela intensidade e tamanho do halo de precipitação formado ao redor dos inóculos.

Atividade da DNase. As bactérias crescidas em ágar LB (Neogen) foram inoculadas através de uma estria linear sobre o ágar DNase (Neogen). As placas foram incubadas a $37^{\circ} \mathrm{C}$ por 18 a 24 h. A solução aquosa de $\mathrm{HCl} 1 \mathrm{~N}$ foi adicionada sobre o crescimento bacteriano e a formação de uma zona clara ao redor da cultura indicou reação positiva para DNase [22].

\subsection{Análise estatística}

Todos os experimentos foram conduzidos em triplicata em ao menos três ocasiões distintas. Para realizar a representação gráfica e a análise estatística foi utilizado o Software R Studio (1.2.5001). Valores de $\mathrm{p}<0,05$ foram considerados como significantes. 


\section{RESULTADOS E DISCUSSÃO}

Neste estudo foram analisados fenotipicamente os fatores de virulência de 19 isolados de Pseudomonas aeruginosa obtidos de água de abastecimento público. Estes isolados foram previamente estudados quanto ao perfil de sensibilidade aos antibióticos e formação de biofilme por Anversa et al. (2019) [2], os quais constataram que as cepas isoladas se mostraram sensíveis a todos os antimicrobianos, exceto ticarcilina/ácido clavulânico, ao qual a resistência foi indicada como intermediário. Também observaram a forte capacidade de produção de biofilme em $10(52,63 \%)$ isolados testados, $7(36,84 \%)$ como produtores moderados e fracos, e apenas 2 $(10,53 \%)$ foram classificados como não produtoras de biofilme.

Com a finalidade de avaliar a ocorrência de fatores de virulência nestes isolados, estes foram submetidos à pesquisa da produção de proteases alcalina, ramnolipídeos, DNases, e dos tipos de motilidade (Tabela 1).

Tabela 1: Expressão fenotípica dos fatores de virulência em isolados de P. aeruginosa utilizados no presente estudo.

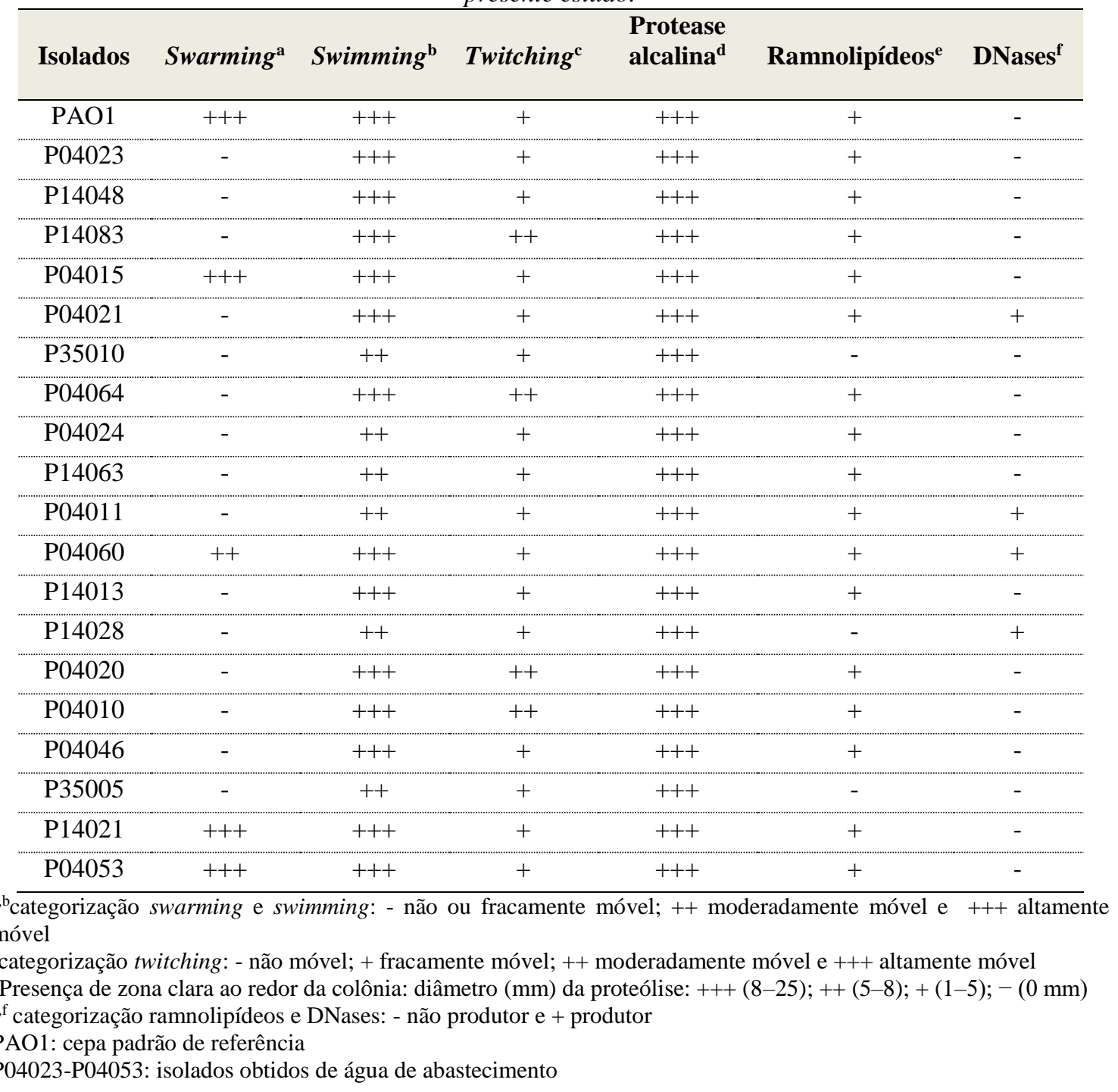

As análises dos fatores de virulência revelaram que todos os 19 isolados de $P$. aeruginosa estudados foram produtores de protease alcalina com zona de proteólise acima de $8 \mathrm{~mm}$. A atividade da DNase e a produção de ramnolipídeos foi observada em 21,1\% (n=4) e 84,2\% $(\mathrm{n}=16)$ dos isolados, respectivamente. As enzimas proteolíticas produzidas por P. aeruginosa contribuem para a multiplicação bacteriana e quebra das defesas físicas do hospedeiro, fornecendo aminoácidos e peptídeos das proteínas teciduais para as bactérias. Além de degradar 
imunoglobulinas e outros componentes interferindo no mecanismo de defesa imunológica do hospedeiro [3]. Os ramnolipídeos auxiliam nos estágios iniciais da formação de biofilmes e tem papel na regulação da motilidade. A atividade da DNase, demonstrou ser responsável pela redução da viscosidade do escarro em pacientes com fibrose cística [11].

Os resultados de motilidade foram interpretados utilizando as medidas dos diâmetros de crescimento, seguidos da categorização. No ensaio de swarming, quatro isolados $(21,1 \%)$ foram positivos, com zona de motilidade variando de 9 a $73 \mathrm{~mm}$, categorizados em: não ou fracamente móvel, 78,9\% ( $\mathrm{n}=15)$; moderadamente móvel, 5,3\% $(\mathrm{n}=1)$; e altamente móvel, 15,8\% $(\mathrm{n}=3)$. No ensaio de swimming, $100 \%(\mathrm{n}=19)$ dos isolados exibiram resultados positivos, com zona de motilidade variando de 21 a $64 \mathrm{~mm}$, sendo 31,6\% (n=6) moderadamente móvel e 68,4\% (n=13) altamente móvel. No ensaio de motilidade twitching as zonas de motilidade variaram de 7 a 17 mm, sendo 78,9\% (n=15) fracamente móvel e 21,1\% ( $\mathrm{n}=4)$ moderadamente móvel. Os resultados mostraram uma alta diversidade no comportamento de motilidade entre os isolados de $P$. aeruginosa. Ao comparar os três tipos de motilidade, a maioria dos isolados $(68,4 \%)$ apresentou produção significativa $(\mathrm{p}<0,05)$ de motilidade swimming (Figura 1).

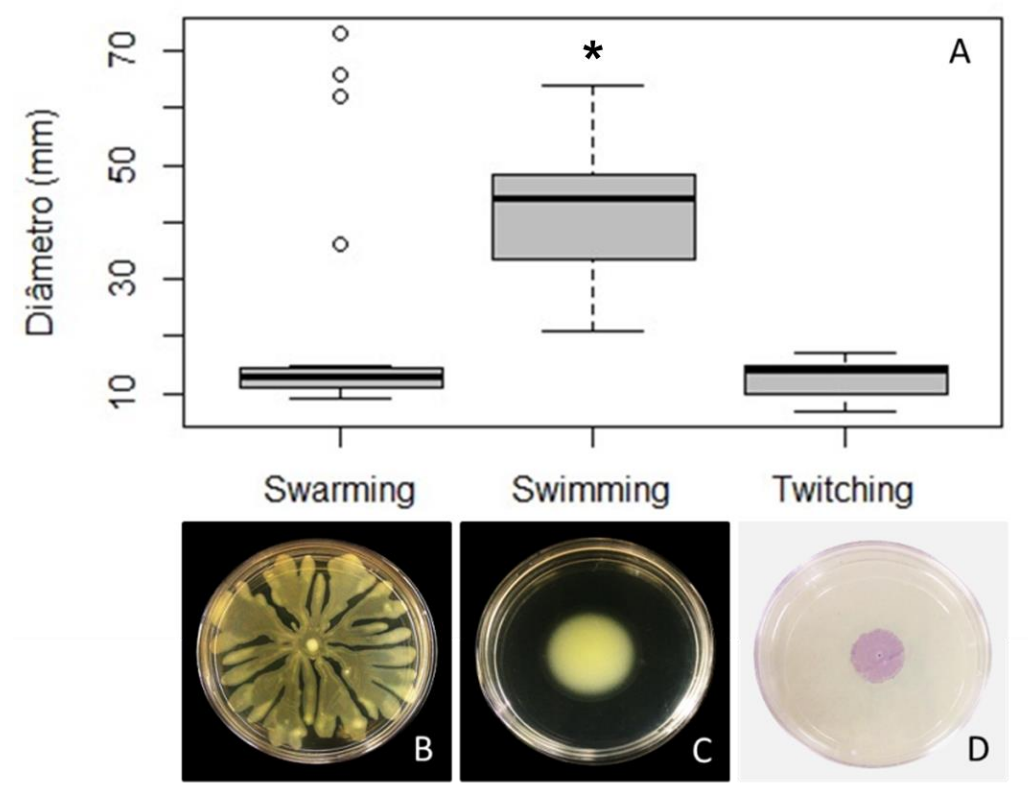

Figura 1: Resultados dos ensaios fenotípicos de motilidade. A) Gráfico box-plot da distribuição de diâmetro das motilidades (mm); B) Motilidade swarming; C) Motilidade swimming; D) Motilidade twitching. * Resultados significativos com $p<0,05$.

Autores como Zineba et al. (2015) [23] relataram que 54,7\%; 43\%; 71\% e 59,5\% dos isolados de $P$. aeruginosa obtidos de água apresentaram produção de protease, motilidade swarming, swimming e twitching, respectivamente. A motilidade swarming formam padrões dendríticos na superfície do meio de cultura, enquanto que em swimming as bactérias movimentam-se de forma desorganizada sobre superfícies aquosas, sendo característica de isolados de ambientes fluidos, como a água [24, 25]. Isso pode estar relacionado às altas porcentagens deste fenótipo no presente estudo. A motilidade twitching ocorre em superfícies sólidas e a única estrutura envolvida é o pili tipo IV. A motilidade bacteriana é essencial para a patogenicidade, uma vez que está envolvida na adesão, colonização, promoção do processo infeccioso, formação de biofilmes e fuga a ambientes desfavoráveis [5, 6, 26].

A virulência e patogenicidade desta bactéria são reguladas pelo Sistema Quorum Sensing (QS), um mecanismo de comunicação celular entre as bactérias através da secreção de sinais químicos extracelulares, e sua concentração é dependente da densidade microbiana [27]. Assim, o aumento da densidade microbiana pode promover o aumento de fatores de virulência como protease alcalina, produção de ramnolipídeos e motilidade, como os encontrados na presente 
pesquisa, e serem potencialmente patogênicas, especialmente para pacientes imunocomprometidos.

A produção de diversos fatores de virulência causa preocupação, devido às suas ações relacionadas ao sucesso invasivo ao hospedeiro, ao transporte pelo meio aquático e, a adesão às superfícies bióticas e abióticas, o que permite sua fixação e sobrevivência em diversos ambientes, incluindo o hospitalar [10, 23].

Em diversos países foram relatados a identificação de P. aeruginosa em ambiente hospitalar relacionando com o sistema de abastecimento de água. Na França, foi identificado que um surto hospitalar por $P$. aeruginosa estava relacionado com a exposição à água potável contaminada [8]. No Reino Unido, pesquisadores identificaram P. aeruginosa em uma torneira do lavatório de uma Unidade de Terapia Intensiva [28]. Na Irlanda do Norte, constatou-se a contaminação das torneiras da Unidade de Terapia Intensiva Neonatal por $P$. aeruginosa, após investigação decorrente da morte de recém-nascidos por bacteremia ocasionada por esta bactéria [29]. Na Itália, os pesquisados identificaram 55,93\% isolados de $P$. aeruginosa em sistema de abastecimento de água do Hospital exibindo diversos fatores de virulência [30]. No Brasil, os relatos da presença de $P$. aeruginosa em águas utilizadas em clínicas de hemodiálise são frequentes $[31,32]$. Desta forma, revela o risco no qual os pacientes renais crônicos estão expostos quando submetidos a este tratamento.

A presença de $P$. aeruginosa nas torneiras foi relacionada à sua capacidade de sobrevivência à baixa exigência nutricional e a capacidade de formação de biofilme, que possibilitam a sobrevivência desta bactéria em superfícies hospitalares podendo ocasionar os surtos de infecção [33]. A combinação de propriedades intrínsecas da bactéria, como as características virulentas e a resistência aos antimicrobianos, as condições ambientais e o estado de saúde dos pacientes são observados como fatores para severidade e alta mortalidade em pacientes com infecções pulmonares e bacteremias [34].

Os resultados deste estudo demonstram a importância de incluir $P$. aeruginosa na avaliação da potabilidade da água, especialmente nas clínicas e ambientes hospitalares. A fim de contribuir para a higienização correta dos sistemas de abastecimento de água dos hospitais, assim como a conscientização dos profissionais da área da saúde sobre as fontes de contaminação por $P$. aeruginosa, além da implantação de medidas para reduzir a disseminação, monitorar o ambiente hospitalar e traçar estratégias para minimizar o risco de contaminação para pacientes suscetíveis $[29,35]$.

\section{CONCLUSÃO}

Conclui-se que a maioria dos isolados de $P$. aeruginosa testados foram capazes de produzir fatores de virulência como motilidade swimming e twitching, protease e ramnolipídeos. $\mathrm{O}$ conhecimento sobre os fatores de virulência destes microrganismos obtidos de águas de abastecimento é essencial para esclarecer a epidemiologia e as possíveis causas de infecções por P. aeruginosa.

\section{AGRADECIMENTOS}

Secretaria da Saúde do Estado de São Paulo (SES/SP).

\section{REFERÊNCIAS BIBLIOGRÁFICAS}

1. Parcell BJ, Oravcova K, Pinheiro M, Holden MTG, Phillips G, Turton JF, Gillespie SH. Pseudomonas aeruginosa intensive care unit outbreak: winnowing of transmissions with molecular and genomic typing. J Hosp Infect. 2018 Mar;98(3):282-288, doi:10.1016/j.jhin.2017.12.005

2. Anversa L, Stancari RCA, Garbelotti M, Ruiz LS, Pereira VBR, Nascentes GAN, Dantas STA, Rall VLM. Pseudomonas aeruginosa in public water supply. Water Pract Technol. 2019 Sep;14(3):732737, doi:10.2166/wpt.2019.057

3. Strateva T, Mitov I. Contribution of an arsenal of virulence factors to pathogenesis of Pseudomonas aeruginosa infections. Ann Microbiol. 2011 May;61(4):717-732, doi:10.1007/s13213-011-0273-y 
4. Ghorbal SKB, Chourabi K, Maalej L, et al. Pseudomonas aeruginosa swarmer cells adaptation toward UVc radiations. Front Microbiol. Apr 2019;10:556, doi:10.3389/fmicb.2019.00556

5. Mattingly AE, Weaver AA, Dimkovikj A, Shrout, JD. Assessing Travel Conditions: Environmental and Host Influences on Bacterial Surface Motility. J Bacteriol. 2018 Mar;200(11):e00014-18, doi:10.1128/JB.00014-18

6. Otton LM, Campos MS, Meneghetti KL, Corção G. Influence of twitching and swarming motilities on biofilm formation in Pseudomonas strains. Arch Microbiol. 2017 Feb;199(5):677-682, doi:10.1007/s00203-017-1344-7

7. Rocha AJ, Barsottini MRO, Rocha RR, Laurindo MV, Moraes FLL, Rocha SL. Pseudomonas aeruginosa: Virulence Factors and Antibiotic Resistance Genes. Braz Arch Biol Technol. Jun;2019; 62: e19180503, doi.org/10.1590/1678-4324-2019180503

8. Costa D, Bousseau A, Thevenot S, Dufour X, Laland C, Burucoa C, Castel O. Nosocomial outbreak of Pseudomonas aeruginosa associated with a drinking water fountain. J Hosp Infect. 2015 Nov;91(3):271-4, doi:10.1016/j.jhin.2015.07.010

9. Garvey MI, Wilkinson MAC, Holden K, Martin T, Parkes J, Holden E. Tap out: reducing waterborne Pseudomonas aeruginosa transmission in an intensive care unit. J Hosp Infect. 2018 May;102(1):7581, doi:10.1016/j.jhin.2018.07.039

10. Galdino ACM, Branquinha MH, Santos ALS, Viganor L. Springer Nature Singapore Pte; 2017. Chapter 16, Chakraborti S, Dhalla N. (eds) Pathophysiological Aspects of Proteases; p. 381-397, doi:10.1007/978-981-10-6141-7_16

11. Das T, Manoharan A, Whiteley G, Glasbey T, Manos J. Pseudomonas aeruginosa biofilms and infections: Roles of extracellular molecules. Elsevier; 2020. Chapter 3, New and Future Developments in Microbial Biotechnology and Bioengineering: Microbial Biofilms; p. 29-46, doi:10.1016/b978-0444-64279-0.00003-7

12. Anghel I, Holban AM, Grumezescu AM, Andronescu E, Ficai A, Anghel G, Maganu M, Lazar V, Chifriuc MC. Modifed wound dressing with phyto-nanostructured coating to prevent staphylococcal and pseudomonal bioflm development. Nanoscale Res Lett. 2012 Dec;7(1):690, doi:10.1186/1556276X-7-690

13. Val AL, Bicudo CEM, Bicudo DC, Pujoni DFF, Spilki FR, Nogueira ISN, Hespanhol I, Cirilo JA, Tundisi JG, Val P, Hirata R, Olievira e Azevedo, SMF, Crestana S, Ciminelli VST. Water quality in Brazil, In: Water quality in the Americas: Risks and opportunities. Inter-American Network of Academies of Sciences IANAS, 2019.

14. Brasil. Portaria Consolidação $n^{\circ} 5$ de 28 de setembro de 2017. Consolidação das normas sobre as ações e os serviços de saúde do Sistema Único de Saúde. Anexo XX - Do controle e da vigilância da qualidade da água para consumo humano e seu padrão de Potabilidade. Diário Oficial da União, Brasília, DF. 2017. 825p.

15. Stoler J, Ahmed H, Frimpong LA, Bello M. Presence of Pseudomonas aeruginosa in coliform-free sachet drinking water in Ghana. Food Control. 2015 Sep;55:242-247, doi:10.1016/j.foodcont.2015.02.038

16. Caskey S, Stirling J, Moore JE, Rendall JC. Occurrence of Pseudomonas aeruginosa in waters: implications for patients with cystic fibrosis (CF). Lett Appl Microbiol. 2018 Jun; 66(6): 537-541, doi:10.1111/lam.12876

17. Norizan SN, Yin WF, Chan KG. Caffeine as a potential quorum sensing inhibitor Sensors. 2013 Apr;13(4):5117-5129, doi:10.3390/s130405117

18. Abdouchakour F, Aujoulat F, Licznar-Fajardo P, Marchandin H, Toubiana M, Parer S, Lotthé A, Jumas-Bilak E. Intraclonal variations of resistance and phenotype in Pseudomonas aeruginosa epidemic high-risk clone ST308: A key to success within a hospital? Int J Med Microbiol. 2018 Mar;308 (2):279-289, doi:10.1016/j.ijmm.2017.11.008

19. Inoue T, Shingaki R, Fukui K. Inhibition of swarming motility of Pseudomonas aeruginosa by branched-chain fatty acids. FEMS Microbiol Lett. 2008 Apr;281(1):81-86, doi:10.1111/j.15746968.2008.01089.x

20. Wolska K, Kot B. Twitching motility activity, biofilm formation, and genetic typing for clinical isolates of Pseudomonas aeruginosa by random amplified DNA PCR. Acta Microbiol Imm H. 2013 Sep;60(3):313-328, doi:10.1556/AMicr.60.2013.3.7

21. Siegmund I, Wagner F. New method for detecting rhamnolipids excreted by Pseudomonas species during growth on mineral agar. Biotechnol Tech.1991 Jul;5(4):265-268, doi:10.1007/BF02438660

22. Macin S, Akarca M, Sener B, Akyon Y. Comparison of virulence factors and antibiotic resistance of Pseudomonas aeruginosa strains isolated from patients with and without cystic fibrosis. Rev Romana Med Lab. 2017 Oct;25(4): 327-334, doi:10.1515/rrlm-2017-0027 
23. Zineba G, Hassan L, Mostafa M, Abdellah H, Mohammed T, Mostafa ME. Virulence phenotype, physicochemical properties, and biofilm formation of Pseudomonas aeruginosa on polyethylene used in drinking water distribution systems. Water Resour. 2015 Jan;42(1):98-107, doi:10.1134/S0097807815010042

24. Yan J, Monaco H, Xavier JB. The Ultimate Guide to Bacterial Swarming: An Experimental Model to Study the Evolution of Cooperative Behavior. Annu Rev Microbiol. 2019 Sep;8(73):293-312, doi:10.1146/annurev-micro-020518-120033

25. Akhtar ST, Sabri AN. Twitching, swimming, swarming in biofilm forming strains in response to chemical and physical factors. Punjab Univ J Zool. 2017 Dec;32(2):265-276.

26. Khan F, Pham DTN, Oloketuyi SF, Kim Y-M. Regulation and controlling the motility properties of Pseudomonas aeruginosa. Appl Microbiol Biotechnol. 2020 Jan;104:33-49, doi:10.1007/s00253-01910201-w

27. Saeki EK, Kobayashi RKT, Nakazato G. Quorum sensing system: Target to control the spread of bacterial infections. Microb Pathog. 2020 Feb;142:104068, doi:10.1016/j.micpath.2020.104068

28. Garvey MI, Bradley CW, Tracey J, Oppenheim B. Continued transmission of Pseudomonas aeruginosa from a wash hand basin tap in a critical care unit. J Hosp Infect. 2016 Sep;94(1):8-12, doi:10.1016/j.jhin.2016.05.004

29. Walker JT, Jhutty A, Parks S, Willis C, Copley V, Turtonr JF, Hoffman PN, Bennett A M. Investigation of healthcare-acquired infections associated with Pseudomonas aeruginosa biofilms in taps in neonatal units in Northern Ireland. J Hosp Infect. 2014 Jan;86(1): 16-23, doi:10.1016/j.jhin.2013.10.003

30. Iseppi R, Sabia C, Bondi M, Mariani M, Messi P. Virulence Factors, Drug Resistance and Biofilm Formation in Pseudomonas Species Isolated from Healthcare Water Systems. Curr Microbiol. 2020 Apr, doi:10.1007/s00284-020-01990-9

31. Figel IC, Dalzoto PR, Pimentel IC. Microbiological quality of water and dialysate from haemodialysis units in Southern Brazil. Rev Inst Adolfo Lutz. 2015 Feb;74(1):66-70.

32. Peresi JTM, Almeida IAZC, Teixeira ISC, Silva SIL, Alves EC, Marques DF, Ribeiro AK. Pseudomonas aeruginosa: ocorrência e suscetibilidade aos agentes antimicrobianos de isolados de amostras de água tratada utilizada em solução de diálise. Rev Inst Adolfo Lutz. 2011 Dec;70(4):542-7.

33. Ahmadi Jalali Moghadam M, Honarmand H, Asfaram Meshginshahr S. Contamination of Hospital Water Supplies in Gilan, Iran, with Legionella pneumophila, Escherichia coli, and Pseudomonas aeruginosa. Interdiscip Perspect Infect Dis. 2015 Sep;2016:1-7, doi:10.1155/2015/809842

34. Cholley P, Thouverez M, Floret N, Bertrand X, Talon D. The role of water fittings in intensive care rooms as reservoirs for the colonization of patients with Pseudomonas aeruginosa. Intensive Care Med. 2008 Aug;34(8):1428-1433, doi:10.1007/s00134-008-1110-z

35. Loveday HP, Wilson J, Kerr K, Pitchers R, Walker JT, Browne J. Association between healthcare water systems and Pseudomonas aeruginosa infections: A rapid systematic review. J Hosp Infect. 2014 Jan; 86(1):7-15, doi:10.1016/j.jhin.2013.09.010 